\title{
Assessing the Spectrum of International Undergraduate Engineering Educa- tional Experiences
}

\section{Dr. Mary E. Besterfield-Sacre, University of Pittsburgh}

Dr. Mary Besterfield-Sacre is an Associate Professor and Fulton C. Noss Faculty Fellow in Industrial Engineering at the University of Pittsburgh. She is the Director for the Engineering Education Research Center (EERC) in the Swanson School of Engineering, and serves as a Center Associate for the Learning Research and Development Center. Her principal research is in engineering education assessment, which has been funded by the NSF, Department of Ed, Sloan, EIF, and NCIIA. Dr. Sacre's current research focuses on three distinct but highly correlated areas - innovative design and entrepreneurship, engineering modeling, and global preparedness in engineering. She is currently associate editor for the AEE Journal.

\section{Dr. Larry J. Shuman, University of Pittsburgh}

Larry J. Shuman is Senior Associate Dean for Academic Affairs and Distinguished Service Professor of industrial engineering at the Swanson School of Engineering, University of Pittsburgh. His research focuses on improving the engineering education experience with an emphasis on assessment of design and problem solving, and the study of the ethical behavior of engineers and engineering managers. A former Senior Editor of the Journal of Engineering Education, Shuman is the Founding Editor of Advances in Engineering Education. He has published widely in engineering education literature, and is co-author of Engineering Ethics: Balancing Cost, Schedule and Risk - Lessons Learned from the Space Shuttle (Cambridge University Press). He received his Ph.D. from the Johns Hopkins University in Operations Research and a B.S.E.E. from the University of Cincinnati. Dr. Shuman is an ASEE Fellow.

\section{Dr. Gisele Ragusa, University of Southern California}

Gisele Ragusa is a Professor of Engineering Education at the University of Southern California. She conducts research on college transitions and retention of underrepresented students in engineering and also research about engineering global preparedness and engineering innovation. She also has research expertise in STEM K-12 and in STEM assessment. She chairs USC's STEM Consortium.

\section{Dr. Cheryl Matherly, The University of Tulsa}

Dr. Cheryl Matherly is Vice Provost for Global Education at The University of Tulsa, where she has responsibility for the strategic leadership of the university's plan for comprehensive internationalization. Dr. Matherly' directs the NanoJapan program, funded by the National Science Foundation in order to expand international research opportunities for students in STEM fields. She is the recipient of two Fulbright grants for international education administrators (Germany and Japan.) She has an Ed.D. in Education Leadership and Culture Studies from the University of Houston.

\section{Dr. Lisa Benson, Clemson University}

Lisa Benson is an Associate Professor of Engineering and Science Education at Clemson University, with a joint appointment in Bioengineering. Her research focuses on the interactions between student motivation and their learning experiences. Her projects involve the study of student perceptions, beliefs and attitudes towards becoming engineers and scientists, and their problem solving processes. Other projects in the Benson group include effects of student-centered active learning, self-regulated learning, and incorporating engineering into secondary science and mathematics classrooms. Her education includes a B.S. in Bioengineering from the University of Vermont, and M.S. and Ph.D. in Bioengineering from Clemson University. 


\title{
Assessing the Spectrum of International Undergraduate Engineering Educational Experiences
}

\begin{abstract}
The National Academy of Engineering together with a growing number of engineering education researchers have underscored the need for U.S. engineering graduates to be capable of effectively collaborating across national boundaries as they "encounter worlds of professional practice that are increasingly global in nature.” Consequently, a necessary first step in this process requires engineering educators and professionals to clearly understand what constitutes a globally prepared engineer and what are the most effective learning experiences to produce such engineers. This paper offers an overview of the progress to date of a NSF funded multiuniversity (Universities A, B, C and D) research initiative that investigates how the various internationally focused learning experiences within engineering (both curricular and cocurricular) impact students' global preparedness.
\end{abstract}

The research protocol involves three phases, broken into separate but interconnected studies. Study One has focused on developing an operational model of international experiences specific to engineering education; establishing constructs of international education and learning outcomes, and then developing a framework that matches these constructs with appropriate assessment instruments. A unique aspect of Study One is the solicitation of expert opinion through a national, comprehensive Delphi exercise. Using the model from Study One, Study Two centers around a mixed-methods study among the four collaborating schools. Here we triangulated measures using two established assessment instruments - the Global Perspective Inventory (GPI) and the Engineering Global Preparedness Index (EGPI) - that each captures different constructs. Statistical methodologies were applied to map outcomes to educational practices, different institutional characteristics, and student backgrounds. We then used the results from these indices to conduct a series of interviews to tease out the underlying experiences that have contributed to each student's global preparedness. These results are informing Study Three, which will involve a much larger cross-institutional quantitative study to analyze the impact of various international experiences, both within and outside the curriculum. Here we will obtain data from a set of 15 different types of engineering programs. We will focus on a wide range of international experiences including military service, living internationally prior to college, or participating in an extended service project, in addition to the more usual short and long-term study abroad programs. The results of and conclusions from the three studies will be disseminated to the larger engineering education community through an innovative, online approach. Not only will we provide this information in actionable forms, but we will also be able to query responders about their own programs, and update the information in near real time. Specifically, we will aggregate information as the various models for developing global preparedness are being employed, and will assess their effectiveness. Results from this initiative offer the engineering education community a set of impactful and flexible research-based globally focused engineering education pedagogical practices that correlate to learning, diverse student populations, and program types.

\section{Introduction}

Engineering and education faculty from four universities have collaborated to conduct a research initiative that examines how the various international education opportunities, both curricular 
and co-curricular contribute to the global preparedness of engineering graduates. The initiative is funded in part by the National Science Foundation, which has concluded that "The frontier challenges of science and engineering are increasingly global. [Therefore] Future generations of the U.S. science and engineering workforce must collaborate across national boundaries and cultural backgrounds, as well as across disciplines to successfully apply the results of basic research to long-standing global challenges such as epidemics, natural disasters and the search for alternative energy sources."1 Clearly, the global preparedness of engineering students is becoming an important educational outcome and is a natural extension to recent concerns by a number of national commissions and scholars, who have also noted the impact of globalization and the implication for continued U.S. economic leadership. ${ }^{2,3,4}$

Hence, the purpose of our collaboration is to comprehensively study the various ways that we can better educate globally prepared graduates given an already crowded curriculum. Specifically, we aim to better understand how the various international experiences both in (curricular) and out (co-curricular) of formal coursework impact students' global preparedness. This research is timely as $21^{\text {st }}$ century engineers are being called upon to solve complex problems in collaborative, interdisciplinary, and cross-cultural contexts. This requires “. . . a new type of engineer, an entrepreneurial engineer, who needs a broad range of skills and knowledge, above and beyond a strong science and engineering background . . "5 Yet, most evidence about how international experiences and education impact engineering students lacks empirical research to guide educational practices. It is only recently that such studies are beginning to appear. 6,7

Engineering faculty have anecdotally recognized that students who participated in study abroad programs tend to develop such skills as problem solving, cross-cultural communication, and working effectively with culturally diverse teams. Living internationally, especially in a nonEnglish speaking country, prepares students to not only take risks, but to learn to adapt to new environments, develop a greater understanding of contemporary issues, and put engineering solutions in a global and social context. ${ }^{8}$ However, further research is required to fully support, quantify, and generalize these findings beyond anecdotal. Stated another way: There is general agreement that international engineering education experiences are beneficial to students, but we don't know empirically the extent that the various experiences contribute to global preparedness, nor do we even agree on what global preparedness is. These experiences are expensive both for the student and for the University that provides the experiences - how can we ensure students are getting an appropriate educational value for their money? How can these experiences be tailored to achieve educational value? How should we advise students based on the individual's background, prior global preparedness, and financial resources so that the experiences are most effective?

This research addresses two perceived gaps in engineering education: 1) the need for a systematic study of curricular and co-curricular offerings in international engineering education to determine the extent to which the various international academic and non-academic experiences impact the global preparedness of engineering students; 2) the identification of the key constructs that characterize a globally prepared engineering graduate. By addressing both, we will contribute to the understanding of how engineering students become globally prepared, while providing educators with important, actionable items about curricular and extracurricular 
practices that can enhance engineering global preparedness. This paper provides an overview to date of a research endeavor that addresses these two concerns.

\section{Overview of the Work}

This project is being conducted by a multidisciplinary team from four universities. Its four major objectives are delineated into three separate, but interconnected studies (i.e., Delphi, mixed-methods, and cross-institutional) combined with a dissemination system, as shown in Figure 1. In carrying out this research, we are testing three primary hypotheses.

- First, that the types of international experiences are correlated with student learning outcomes. That is, the variety of activities and degree of international exposure that engineering students have is positively correlated with global preparedness.

- Second, specific approaches and/or experiences along with content delivery are correlated with student learning outcomes. Specifically, instructional approaches, extracurricular experiences, and student background factors impact the degree to which student learning outcomes are achieved.

- Third, different international activities positively affect the attitudes and preparedness of different engineering student groups (e.g., minorities, women, foreign nationals, veterans). Through modeling efforts the team will connect student learning outcomes directly to educational practices, institutional characteristics, and student factors.

When completed, this project will provide the engineering community with a set of practices correlated with international learning, various student populations, and types of programs. Each study is discussed and an overview of how each question is addressed follows. We draw upon a definition of engineering global preparedness that has emerged from our research. Specifically, an engineering student's global preparedness requires him or her to become aware of, and able to contribute to a global engineering workforce and marketplace. ${ }^{9,10,11,12}$

Study One: Expert Developed Framework. The purpose of Study One has been to establish a baseline model of the global engineer's professional attributes, to expand these attributes to constructs and learning outcomes, and to ultimately develop complementary instruments focused on measuring the outcomes. To do this, the team conducted a comprehensive Delphi study, identifying and then obtaining opinions from experts on: the constructs and the learning outcomes of these constructs based on the initial set of attributes. The Delphi study consisted of three rounds that culminated with a face-to-face meeting followed by a fourth and final analytical and mapping synthesis. The sample included 18 Subject Matter Experts (SMEs) representing engineering faculty with experience in international education, international education practitioners, industry representatives familiar with international engineering assignments, and project officers from agencies that sponsored international engineering opportunities. In Round 1 participants addressed two open-ended questions:

First, what characterizes a globally prepared engineer?; and,

Second, what are the learning experiences necessary to produce such an engineer?

Their responses were used to construct a questionnaire that participants completed in the second round. From the participants' second round responses areas of consensus and divergence were 
identified and used for the third round, in which the SMEs revised their judgments and provided their rationale. These were then discussed at a face-to-face "summit," at which participants came to consensus about the learning outcomes and programmatic elements that influenced the quality of global experiences, and addressed the connections to global preparedness. As part of the summit, the SMEs created semantic maps of global engineering preparedness outcomes. Following the summit, these were then synthesized into a single map that was vetted by the SMEs during the fourth and final round. The resulting map (see Figure 2) provides an organizing framework for international engineering education and illustrates the interrelationships among engineering global preparedness attributes and three other broad categories: intercultural contextual knowledge, personal and professional qualities, and cross-cultural communication skills and strategies. The outcomes from this study were used to produce a model of global engineering preparedness, which helped to provide the basis for a student background instrument that was employed in Study Two, as well as provided a means to determine how certain outcomes were achieved from students' international and global experiences.

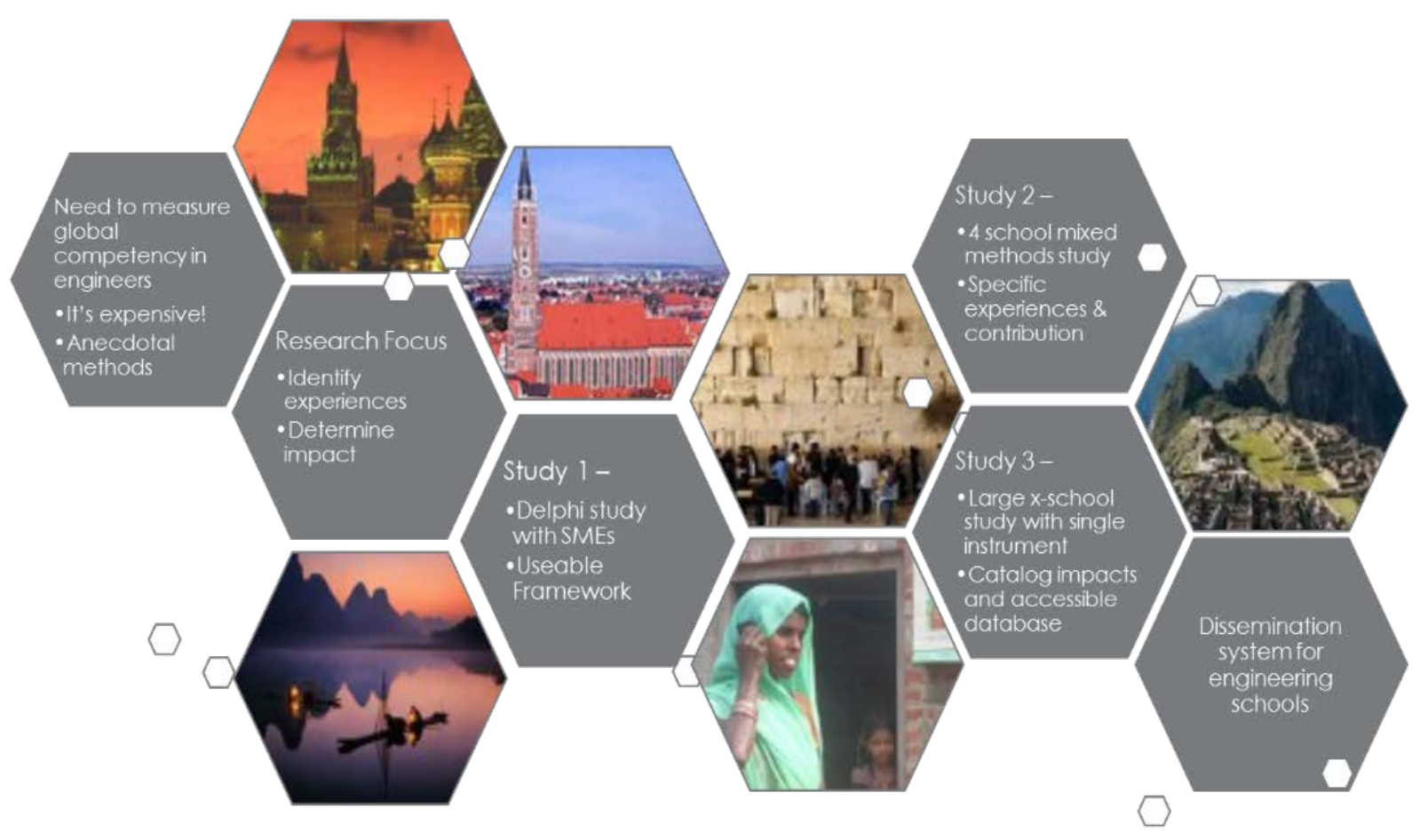

Figure 1. Overview of the Research Agenda

Study Two: Mixed Methods Experiment. This study uses a mixed-methods quasi-experimental design to measure the learning outcomes identified in Study One. Learning outcomes are obtained through a mapping of the constructs with the two instruments' (EGPI and GPI) scales discussed below.

The Global Perspective Inventory (GPI). The GPI, developed by Braskamp, Braskamp, and Merril, is anchored by two theoretical perspectives grounded in holistic human development: intercultural maturity (e.g. trying to make sense of their journey through life) and intercultural 
communication (e.g. the thinking, feeling, and relating domains) ${ }^{13}$. The GPI has been administered at over 150 institutions; over 100,000 students, staff, and faculty have completed the GPI since its development in $2008^{13}$. The instrument draws on the work of Kegan, who argued that as people grow, they are engaged in meaning making ${ }^{14}$. It identifies three major domains of human development and associated questions, as delineated in Table 1.

\section{Table 1: GPI Sample Items by Selected Subscales/Constructs}

\begin{tabular}{ll}
\hline $\begin{array}{l}\text { Subcale/Construct } \begin{array}{l}\text { Cognitive } \\
\text { Knowing }\end{array} \\
\begin{array}{l}\text { Intrapersonal } \\
\text { Identity }\end{array}\end{array}$ & $\begin{array}{l}\text { I take into account different perspectives before drawing conclusions } \\
\text { about the world around me. }\end{array}$ \\
\hline $\begin{array}{l}\text { Intrapersonal } \\
\text { Affect }\end{array}$ & I am sensitive to those who are discriminated against. \\
\hline $\begin{array}{l}\text { Interpersonal } \\
\text { Social Interaction }\end{array}$ & $\begin{array}{l}\text { I frequently interact with people from a race/ethnic group different from } \\
\text { my own. }\end{array}$ \\
\hline
\end{tabular}

The first domain is cognitive. This domain considers the question, "How do I know?" It is centered on one's knowledge and understanding of what is true, what is important to know, and how one determines each of these things. This domain includes the subscales of Knowing and Knowledge. Knowing is the degree of complexity of one's view of the importance of cultural context in judging what to know and value. Knowledge is the degree of understanding and awareness of various cultures and their impact on our global society; it is also the level of proficiency in more than one language. The second domain is intrapersonal. It asks "Who am I?” and seeks to understand how one integrates one's personal values and self-identity into one's personhood and how one becomes more aware of this process. The Intrapersonal domain consists of the Identity and Affect subscales. Identity is a combination of the level of awareness of one's unique identity and degree of acceptance of one's ethnic, racial, and gender dimensions of that identity. Affect is the level of respect for and acceptance of cultural perspectives different from one's own and degree of emotional confidence when living in complex situations. Finally, the third domain is interpersonal social interaction. This domain asks "How do I relate to others?"; and considers one's willingness to interact with persons with different social norms and cultural backgrounds, acceptance of others, and comfort with relating to others. The Interpersonal domain consists of Social Responsibility and Social Interactions subscales. Social Responsibility measures the level of interdependence and social concern for others. Social Interactions measures the degree of engagement with others who are different from oneself and degree of cultural sensitivity when living in pluralistic settings.

The Engineering Global Preparedness Index (EGPI). The EGPI is aligned to both ABET's more difficult to measure professional skills and the NAE's, Engineer of 2020. The EGPI is not a survey of perception of learning; rather, it directly measures how prepared students are for the global workforce. The index is grounded in global citizenry theory. ${ }^{15,16}$ It utilizes four subscales, as provided in Table 2, each of which have been validated using item response theory ${ }^{17}$ and extensively tested for reliability. 
The first subscale is Global Engineering Ethics and Humanitarian Values. This construct refers to the depth of concern for people in all parts of the world, with a view of moral responsibility to improve life conditions through engineering problem solving and to take such actions in diverse engineering settings. The second subscale is Global Engineering Efficacy. This refers to the belief that one can make a difference through engineering problem solving and is in support of one's perceived ability to engage in personal involvement in local, national, international engineering issues and activities towards achieving greater global good using engineering methodologies and approach. Engineering Global-centrism is the third subscale. This refers to a person's value of what is good for the global community in engineering related efforts, and not just one's own country or group. It refers to one's ability to make sound judgements based on global needs in which engineering and associated technologies can have impact on global improvement. Finally, Global Engineering Community Connectedness is the last subscale. This subscale refers to one's awareness of humanity and appreciation of interrelatedness of all people and nations and the role that engineering can play in improving humanity, solving human problems via engineering technologies, and meeting human needs across national boundaries.

\section{Table 2: EGPI Sample Items by Selected Subscales/Constructs}

\begin{tabular}{ll}
\hline $\begin{array}{l}\text { Subcale/Construct } \\
\begin{array}{l}\text { \& Humanineering Ethics } \\
\text { Values }\end{array}\end{array}$ & $\begin{array}{l}\text { Sample Index Item } \\
\text { engineers in my country have a moral obligation to share their }\end{array}$ \\
\hline $\begin{array}{l}\text { Global Engineering } \\
\text { Efficacy }\end{array}$ & $\begin{array}{l}\text { I believe that my personal decisions and the way that I implement them } \\
\text { in my work activities can affect the welfare of others and what happens } \\
\text { on a global level. }\end{array}$ \\
\hline $\begin{array}{l}\text { Engineering } \\
\text { Global-centricism }\end{array}$ & $\begin{array}{l}\text { I think my country needs to do more to promote the welfare of different } \\
\text { racial and ethnic groups in engineering industries. }\end{array}$ \\
\hline $\begin{array}{l}\text { Engineering } \\
\text { Community } \\
\text { Connectedness }\end{array}$ & $\begin{array}{l}\text { To treat everyone fairly, we need to ignore the color of people’s skin in } \\
\text { our workplaces. }\end{array}$ \\
\hline
\end{tabular}

An accompanying background survey was developed to identify those underlying student characteristics and the student's respective international/intercultural experiences contributed most to the individual's global preparedness as identified in Study One. The resultant background survey instrument consisted of four components: profile characteristics (e.g., gender, age, class standing,), educational background (e.g., university, major, QPA), travel abroad/ international experiences (e.g., level of interest in international issues, foreign language proficiency), and characteristics of the international experiences (e.g., programmatic elements of experiences such as duration, amount of reflection, and comfort zone). The background survey items also provided independent predictor variables to help explain the results of the outcome instruments (EGPI and GPI). Samples (from each of the four partner institutions) of senior engineering students, each of whom had engaged in an at least one international experience were invited to complete the set of instruments (EGPI, GPI, and background survey). In addition to sampling seniors with international experiences, each campus also recruited a comparison set of senior engineering students who had not had an academic-based international experience, and a 
third sample of incoming freshmen, the former serving as a comparison group and the latter providing an institutional baseline.

For the initial data collected in the Spring 2014, descriptive statistical analyses were conducted as well as common comparison tests (i.e., $t$-tests and Cohen's $d$ ). To determine which types of experiences and their programmatic elements predicted learning outcomes related to global preparedness (GPI/EGPI scores), stepwise regression techniques were executed using SPSS, Version 22. In this study, stepwise regression was used to determine relationships between learning experiences and participating students' GPI and EGPI scores (dependent variables). Separate regressions were conducted for each dependent variable. Such analysis will be repeated with the additional data collected in the Fall 2014 and Spring 2015, and structural equation modeling will be further applied to identify those background variables and experiences that are key to global preparedness.

After initially analyzing the results of the EGPI and the GPI, a small subset of students were invited to participate in a follow-up interview of 20-30 minutes. Specifically, we have been interviewing students at each institution who "scored" high on the outcome instruments (EGPI and GPI). The primary purpose of these follow-up interviews is to further tease out the underlying reasons for how these students' achieved relatively high levels of global preparedness. The following overarching questions framed the interviews: "Why did you choose to study abroad and/or pursue an international experience?”, "Did these experience change the way you think about engineering?", and "Did these experiences affect your thinking about the cultural relevance of engineering?” A set of probes based on the constructs of the two instruments and background survey helped to facilitate the overarching questions.

An inductive coding protocol was utilized to further refine definitions while allowing for additional analytical themes to emerge. The preliminary codes consisted of the type of international experience and structure, motivation, openness to experience, degree of reflection. The transcripts were then coded according to a final schema by multiple research team members to ensure inter-rater reliability, and arbitrated where necessary. Several themes emerged, three of which are pertinent to our research.

Study Three: Cross Institutional Study. Parkinson has identified 24 exemplar engineering schools that promote international education. In Study Three (planned for Fall 2015) we will further test the hypotheses by inviting a representative sample of 12 to 15 engineering schools to participate in an in-depth study to analyze engineering students' global preparedness as the result of their academic and non-academic international experiences. With the completion of Study Two, the team will have an improved, finalized student background instrument; definitive factors linked to global preparedness will have been identified, validated, and incorporated into the background instrument. The research team will use this instrument in conjunction with most likely only one of the outcome instruments - the EGPI or GPI. The purpose of the third study is to further test and explore the three hypotheses and findings from the second study.

Dissemination Platform. Both traditional and innovative means for dissemination will be used. We will leverage the extensive networks that we have developed (and will be developing) to cultivate an influential group of users for distributing research results, engaging them in both development and dissemination activities. In particular, we propose a creative way to both disseminate our results to a wide spectrum of engineering programs, while at the same time 
asking them to assist us in further extending our data base and findings. In this manner we anticipate obtaining a more comprehensive picture of the various international educational experiences provided by U.S. engineering schools. At the completion of Study Three we will have a well-defined framework, having brought an additional 12 to 15 schools into the project. Using data obtained from these schools as described above, we will have constructed models, validated through interviews, that will better enable us to identify those factors, including various pedagogical approaches, and formal and informal educational formats that lead to global preparedness. This will be organized in a manner that will allow translation into practice for engineering administrators and faculty as they consider how best to prepare their students for the global economy. Concomitantly, we will systematically utilize available resources including the web to determine the global opportunities that each U.S. engineering school offers its undergraduates, including the range of study abroad experiences, co-op and internship opportunities, and service learning experiences through active chapters of organizations such as Engineers without Borders and Engineers for a Sustainable World.

\section{Lessons Learned To Date}

We are currently at the mid-point of our research agenda. We have completed Study One and have finished our data collection and initial analysis for Study Two. This section provides a summary of the lessons learned at this point in the project.

Study One: Expert Developed Framework. As described above, the first study involved a threeround Delphi experiment with subject matter experts (SMEs). It concluded with a summit that brought the SMEs together with the research team. The Summit involved a series of structured activities including a review of the construct groupings generated from the Delphi questionnaire in order to better achieve consensus regarding the clustering/aggregating of global preparedness outcomes, and a mapping exercise. Summit participants also engaged in discussions about measures of quality of international experiences from the Delphi study the context of achieving engineering global preparedness.

In addition, SMEs participated in developing a conceptual model of global preparedness. Although only 12 of the 18 SMEs were able to participate in the summit, the initial data to develop the maps was generated from all 18 SMEs during the first three rounds of the Delphi study. The participating SMEs were divided into four sub-groups for the semantic mapping exercise. The groups were asked to create semantic maps reflecting major constructs of engineering global preparedness using data collected from the Delphi processes and to indicate relationships among constructs or categories.

A synthesized semantic map was generated by the research team from the four semantic maps based on the similarities of those items whose degree of consent was two or larger. This synthesized map was provided post-summit to all 18 SME participants for feedback. The feedback then was incorporated into a final conceptual model. The culminating semantic map resulting from this analysis formed a conceptual model of global engineering preparedness, which compares favorably to those reported in the literature. Figure 2 illustrates the final results of the conceptual model. The model depicts relationships among engineering global preparedness, international contextual knowledge, personal and professional qualities, and cross-

cultural communication skills and strategies. ${ }^{18}$ In summary, through the three Delphi rounds and 
summit mapping experience, the SMEs provided a full set of learning experiences that prepare students for global engineering workforce.

The second critical outcome of the first study was the SME's expansive weighted list of experiences and the constructs that define the quality of those experiences. These two results from Study One provide a solid understanding of what global preparedness means relative to engineering education, as well as the foundation to measuring the impact that the various students' experiences may have had. Further, the collection of experiences, their constructs, along with the outcomes provides a useful structure for those faculty who are tasked with designing new international engineering education programs. ${ }^{19}$ Both of these products provide value on their own but were critical in the development of Study Two. Hence, two primary products resulted from this phase of the research.

Study Two: Mixed Methods Experiment. We have recently concluded the data gathering portion of our mixed methods experiment. Our sample includes data from 183 participants from the spring 2014 implementation of our experiment from three of the four institutions. Our analysis to date indicates that students' experiences have varied greatly. Perhaps not surprising, more than $70 \%$ of these respondents who had one or more international experiences described high to extremely high personal interest in international issues and learning about other cultures. More than half of the participants indicated that they had resided in or visited three or more countries in their lifetimes and approximately one-third of the participants had lived in or traveled to one or two countries. On the other hand, $14 \%$ of the respondents reported that they had never been abroad, even though they had scored high on the outcome measures.

With regard to globally or internationally focused course work, the majority of students in the sample completed approximately three college-level courses that focused on global, crosscultural, intercultural or international content; and spent, on average, one-third of their time working with non-US students in their classes. However, 74\% of the respondents said they did not have fluency in a foreign language.

To determine relative differences and similarities in student performance across the two outcome measures (the EGPI and GPI) independent sample $t$-tests were calculated. Participation in experiences such as study abroad, engineering/non-engineering focused service learning, engineering/non-engineering courses with global focus, and personal tourism abroad are statistically relevant to students' performance on both the GPI and EGPI. As hoped, those who participated in international or globally focused experiences outscored those who did not. Notably, the mean EGPI score of students who reported study abroad was significantly higher than that of those who did not study abroad. In contrast, participation in second-language courses, projects or internships abroad, or having an international roommate did not reveal a statistically significant difference in students’ EGPI or GPI performance.

Stepwise regression analysis was used to determine potential relationships among student experiences and their global preparedness. The regression results indicated that the combination of such experiences including engineering focused service learning, study abroad, and nonengineering course(s) with a global focus accounted for approximately $12 \%$ of the variance in the GPI rating. These three types of experiences contributed to model fit $(\alpha=0.05)$, with study abroad being the major contributor, followed by an engineering focused service learning program, and non-engineering course with a global focus. Using the EGPI as the outcome 
measure in the regression, a combination of engineering focused service learning program, nonengineering focused service learning, engineering course with a global focus, and personal tourism accounted for $17.5 \%$ of the variance in the overall score. These experiences contributed significantly to the model $(\alpha=0.05)$ with personal tourism being the strongest contributor, followed by non-engineering focused service learning, engineering course with a global focus, and engineering focused service learning. ${ }^{20}$ See reference 20 for additional insights.

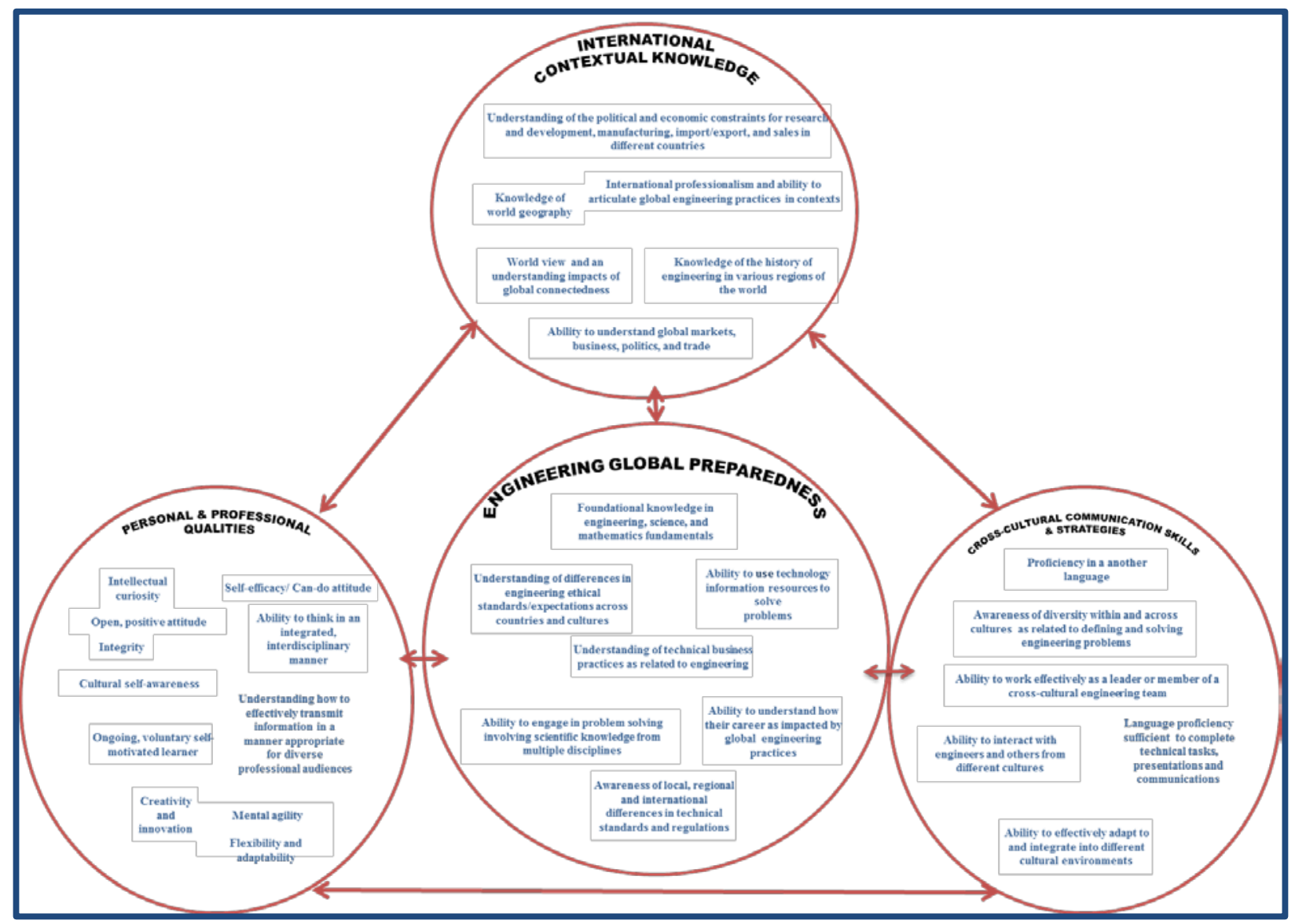

Figure 2. Operational model (semantic map) for engineering global preparedness

In addition to our preliminary quantitative analyses, several themes emerged from the one-onone interviews and the resulting narratives. Three of these themes were particularly prevalent across the student interviews: The Role of Student Expectations, The Mechanisms for Meaningful International Experiences, and The Impact of 'Engineering' on Engineering Experiences Abroad. In addition, two other themes occurred but with less frequency: Perceived Insightfulness and Novelty of the International Engineering Education experience. However, it should be noted that this analysis has been based on data collected during the spring 2014 term and does not include all interviews conducted for the study; hence, the emerging themes are still preliminary. ${ }^{21}$ The three main themes that emerged are discussed below.

The Role of Student Expectations: Based on the interview results, students emphasized what they hoped to gain from an experience and how it related to what they ultimately reported that they brought back from the experience. What a student learned from an international experience was 
motivated in large part by his or her expectations prior to the experience. That is, students' learning outcomes and how they created meaning from their experiences were often dependent on what they wished to gain from the experience. International experiences did not necessarily alter a student's expectations or worldview, but rather, the international experience often helped to confirm his or her expectations. From the interviews to date, an engineering student will get the most meaningful experience and be more globally prepared, independent of background, if he or she can clearly identify expectations, and be able to make sense of the relationship between these expectations and the international environment in which they are asked to participate.

The Mechanisms for Meaningful International Experiences: The second theme that emerged from the interviews related to the elements of an international experience that were most important for an engineering student and his or her ability to develop into a more globally prepared engineer. Much of what the students focused on was the structure of the international experience rather than the content of the program. Specifically, those students who demonstrated the highest levels of global preparedness, pointed to solo immersion and duration as key programmatic components of their meaningful international experience. As one might anticipate, duration, specifically longer (semester to year long) international experience was also indicated by many high scoring students as being vital to a meaningful international experience. These results directly relate to our findings from Study One, as our SMEs specifically directed length of time and immersion as key features of a quality experience. Seriously engaging with the local culture, whether the interaction is purposeful or serendipitous was a common theme noted in most student interviews. This was another aspect that the SMEs from Study One pointed to as a quality feature of an international experience.

An additional component of this theme was the idea that an international engineering experience in which a student has had the opportunity to help others was meaningful and valuable in the development of global preparedness. However, most participants discussed helping international communities through non-engineering means, becoming more aware of the constraints and obstacles that are required to make a difference, and realizing there is more to international development and service than engineering. Experiences that integrated service in some capacity, and allowed students to have meaningful interactions with the host culture and local populace, as well as being sufficiently long to enable the student to become immersed in authenticity appear to be very meaningful to engineering students, and may be vital to the development of global preparedness for future generations of engineers.

The Impact of 'Engineering' on Engineering Experiences Abroad: The students in the study reflected on a wide spectrum of topics during the interviews about their international experience. They spoke about what they learned, or what motivated them to pursue such experiences. The students were also somewhat specific about the events that were the most meaningful, or the stereotypes that were broken down from an increased amount of cultural exposure. However, one topic that was absent from the interview study data was "engineering." While there were a few students who reflected on the engineering aspects of their study abroad experiences in detail, the majority of student interviewed (both high and low scorers in global preparedness), did not connect engineering with deeper values and cultural assumptions. Moreover, the students' posited "meaningful” experiences often did not reflect direct reference to the engineering aspects of the trip, but focused more on its socio-cultural aspects. In many cases, the students preferred to have their international experience not focus on technical engineering aspects. For example, 
some students reflected and learned more about how to work in engineering groups and how to communicate more effectively, as opposed to the engineering work itself. This may be because of the difficulty of creating a true, outside-the-classroom engineering experience in an international setting, but it is somewhat surprising for those who specifically had an engineering focused study abroad experience.

In addition, there was limited emphasis on technical engineering skills in the student interview responses. The research team presented a conceptual map of engineering global preparedness from Study One, ${ }^{22}$ to each student and had him or her reflect on which parts of the map resonated in regards to his or her international experience. There was a general mix of crosscultural communication skills and strategies, personal and professional qualities, and international contextual knowledge (entirely dependent on the type of experience), but engineering global preparedness, the centerpiece of the model, was consistently regarded as “unimportant”, "least relevant”, or ignored.

\section{Current and Future work}

As mentioned, we have just concluded our data collection for Study Two and intend to conduct a full analysis of both the quantitative and qualitative data. In addition to more traditional statistical methods (e.g., hypothesis testing multiple regression), structural equation modeling approach as well as a multivariate analysis of covariance (MANCOVA) model will be used to explore relationships among variables and to determine potentially predictive values of the independent on dependent variables. In this aspect, our investigation builds on the work of a previous study in global preparedness, using a similar design. ${ }^{23,24,25}$ Our instrument outcome measures (EGPI and GPI) will serve as the primary measurement for our research. Our initial multivariate model will include all independent variables, as we intend to explore relationships among the variables. We have primarily designed our model recognizing that these variables may change, but it will provide both an example equation and a starting point for this multivariate analysis.

As we have described above, Parkinson identified 24 exemplar engineering schools that promote international education. ${ }^{26}$ Given this list, a list of potential schools based on an analysis of U.S. News and World Report top 20 lists, and the assistance of the Global Engineering Education Exchange (GE3), we will further test our hypotheses by inviting 15 engineering schools to participate in an in-depth study (Study Three) to analyze engineering students' global competency gained from academic and non-academic international experiences. Study Two will provide insights on how to improve and finalize the important student background instrument with definitive factors that are linked to global preparedness. We will use this instrument in conjunction with our outcome variables on this cross institutional sample of programs.

Recruitment of schools is currently underway and data collection will commence fall 2015. More comprehensive models and tested hypotheses will result from this phase of work and provide a foundation for dissemination.

\section{Summary}

Approximately 500,000 students are enrolled in U.S. engineering programs, now producing over 80,000 B.S. engineering graduates annually. An increasing percentage of graduates are employed in international environments. Further, the demand for globally prepared engineers will continue 
to increase; faculty and administrators will need to offer opportunities to acquire such skills, knowledge, and mindset. This study will provide key data and tools to facilitate the development and improvement of educational opportunities to stimulate international education among undergraduate engineers. Faculty will have access to information on what is typically taught and how institutional and course factors can impact positive student learning outcomes. Although this research focuses more specifically on engineering, the results will be generalizable to other STEM disciplines attempting to improve the global competencies of their students.

\section{Acknowledgements}

This research is being funded by the National Science Foundation, "Collaborative Research: Assessing the Spectrum of International Undergraduate Engineering Educational Experiences” (REE/EEC - 1160404). Portions of this paper were previously presented in ASEE conference proceedings (2013, 2014).

\section{References}

1. National Science Foundation, Investing in America's Future: Strategic Plan, FY 2006-2011 (Arlington, VA: National Science Foundation).

2. Lieberman, J. (2004, May 11). Offshore outsourcing and America's competitive edge: Losing out in the high technology R\&D and services sectors. Washington, DC: U.S. Senate, Office of Joseph I. Lieberman. [White paper] (Retrieved on-line on January 9, 2008 from http://lieberman.senate.gov/documents/whitepapers/Offshoring.pdf).

3. National Science Board. (2004). Science and engineering indicators 2004 (Volume 2, NSB 04-1A). [Electronic version]. Arlington, VA: National Science Foundation (Retrieved on-line on January 5, 2008 from http://www.nsf.gov/statistics/seind04/pdfstart.htm).

4. National Academy of Engineering. (2004). Assessing the capacity of the U.S. engineering research enterprise. (Retrieved on-line on January 8, 2008 from http://www.nae.edu/nae/engecocom.nsf/weblinks/MKEZ68HQMA?OpenDocument).

5. Jonassen, D., Strobel, J., \& Lee, C. B. (2006). Everyday problem solving in engineering: Lessons for engineering educators. Journal of Engineering Education, 95(2), 139-151.

6. Jesiek, B. K., Haller, Y., \& Thompson, J. (2014). Developing Globally Competent Engineering Researchers : Outcomes-Based Instructional and Assessment Strategies from the IREE 2010 China Research Abroad Program. Advances in Engineering Education, 4(1), 1-31.

7. Jesiek, B. K., Thompson, J., \& Mazzurco, A. (2014). Global Engineering Competency in Context : Situations and Behaviors Global. Online Journal for Global Engineering Education, 8(1).

8. Machotka, M. and S. Spodek (2002). "Study Abroad: Preparing Engineering Students for Success in the Global Economy,” (CD) Proceedings, 2002 American Society for Engineering Education Conference.

9. Besterfield-Sacre, M., Matherly, C., G. Ragusa, L. Howard, and L.J. Shuman. "Assessing the Spectrum of International Undergraduate Engineering Education Experiences,” 2013 ASEE International Forum, Atlanta, GA, June 22, 2013.

10. S. Huang, S. Levonisova, Streiner, S., S. Cunningham, G. Ragusa, M. Besterfield-Sacre, L. Shuman, C. Matherly, and D. Kotys-Schwartz, "Exploring Engineering Education in Broader Context: A Framework of Engineering Global Preparedness,” 2014 ASEE Annual Conference and Exposition, Indianapolis, IN June 2014.

11. Streiner, S., S. Cunningham, S. Levonisova, S. Huang, C. Matherly, M. Besterfield-Sacre, G. Ragusa, L. Shuman, and D. Kotys-Schwartz, Moving Toward a Research Informed Conceptual Model of Engineering Global Preparedness, 2014 ASEE Annual Conference and Exposition, Indianapolis, IN June 2014 
12. Streiner, S., M. Besterfield-Sacre, L.J. Shuman, and K. Bursic, "An Approach to Evaluate Engineering Global Preparedness in Industrial Engineering Curricula," 2014 Industrial and Systems Engineering Research Conference (ISERC), Montreal, Canada, May 31 - June 3, 2014.

13. Braskamp, L. A., D. C. Braskamp, \& M. Engberg (2013). Global Perspective Inventory. Global Perspective Institute, Inc., http://gpi.central.edu

14. Kegan, R. (1994). In Over Our Heads: The Mental Demands of Modern Life. Cambridge, MA: Harvard University Press.

15. Zeichner,K.2009. Teacher education and the struggle for social justice. New York: Routledge.

16. Banks, J. 2004 Teaching for Social Justice, Diversity and Citizenship in a Global World."The Educational Forum, 68, 296-305.

17. Yin, 2003 Case Study Research. Thousand Oaks, Sage.

18. Ref. 11.

19. Ref. 10.

20. Levonisova, S., R. Savage, Streiner, S., E. McCave, G. Ragusa, C. Matherly, L. Benson, M. Besterfield-Sacre, and L. Shuman, Identifying Factors that Enhance Undergraduate Engineering Students' Global Preparedness,” 2015 ASEE Annual Conference and Exposition, Seattle, WA June 2015

21. Streiner, S., E. McCave, S. Levonisova, R. Savage, M. Besterfield-Sacre, G. Ragusa, L. Benson, C. Matherly, and L. Shuman, "An Inductive Qualitative Analysis of Student Interviews on Engineering Global Preparedness,” 2015 ASEE Annual Conference and Exposition, Seattle, WA June 2015

22. Ref. 11.

23. Ragusa, G. (2010) Preparing University Students For Global Workforces: Comparisons Between Engineering and Business School Students. Conference Proceedings: Annual Meeting American Society of Engineering Educators, Louisville: KY. June, 2010.

24. Ragusa, G. (under review) Engineering Global Preparedness: Parallel Pedagogies, Experientially Focused Instructional Practice. Submitted to International Journal of Engineering Education.

25. Ragusa, G. and C. T. Lee (under review) The Impact of Focused Degree Projects in Chemical Engineering Education on Students' Achievement, and Efficacy. Submitted to Journal of Chemical Engineering Education.

26. Parkinson, A. (2007). "Engineering Study Abroad Programs: Formats, Challenges, Best Practices,” Online Journal for Global Engineering Education, 2(2). 